\title{
Detection of a Tornado Event on 31 March 2019 and Its Effects on the Eastern Part of Nepal
}

\author{
Tek Bahadur Chhetri 1,2, Binod Dawadi 1,3,*, Yam Prasad Dhital ${ }^{4}$ \\ ${ }^{1}$ Central Department of Hydrology and Meteorology, Tribhuvan University, \\ Kathmandu, Nepal; tekkckc@gmail.com, dawadib@cdhmtu.edu.np \\ 2Institute of Tibetan Plateau Research, Chinese Academy of Sciences, Beijing, China \\ ${ }^{3}$ Kathmandu Center for Research and Education, \\ Chinese Academy of Sciences-Tribhuvan University, Kathmandu, Nepal \\ 4Society of Hydrologists and Meteorologists (SOHAM), Kathmandu, Nepal; ypdhital@gmail.com
}

\begin{abstract}
Objectives: To discuss and introduce the reality of the first tornado in Nepal: its development process, position, shape and size, and power strength. Methods: We analysed data of satellite images from 30 to 31 March 2019, which was downloaded from EOSDIS NASA - a world weather archive system of daily weather and climate observations that is freely available over the Internet. Various true colour images of Terra Modis, with corrected reflectance, were observed to detect the cloud cell thickness by the use of cloud density with brightness temperature images that provide the colour difference both in day and nights, which can be observed very easily. Findings: From the analysis of test image samples, the black-white background of cloud mask system of brightness temperature that clearly distinguished the cyclonic structure of the tornado with its shape, size, and position including its tracks in the atmosphere was found, which was also compared with the image from European satellite systems to conform its real power strength. We found large instability of the atmospheric condition with large heating system of surface temperature and solar radiation with the two systems of air mass (Westerly and S-Easterly) transports combined each other and produced the environment for the formation of more powerful tornado on 31 March 2019 in Nepal. Many atmospheric cyclonic activities in different climatic conditions and their impacts were observed in the past; however, recent cyclone-induced hazards are assumed to be increasing because of regional weather and climate change in south Asian countries. Even a single weather event, first tornado in Nepal on 31 March in the south-eastern part of Nepal, made large-scale damages of people and settlements within a few hours. Application: Many freely available Internet systems for observing extreme event of weather and climatic conditions can be used for daily forecasting system in order to ensure safety from all possible disasters.
\end{abstract}

Keywords: Weather-change, Tornado, Impacts, Sat-image, Forecasting, South Asia

\section{Introduction}

Tornadoes are formed when a large convection of atmospheric disturbance exists due to large unstable condition of atmosphere with respect to heat, temperature, low pressure, and high wind speed. Basically, tornadoes are formed when moist vapour mass transport to atmosphere, where the large mass of vapour condense due to high temperature lapse rate on its ascent or transport path. In this situation, a vast amount of heat is released, which cause temperature inversion (warming) in higher level atmospheric layer and heated up. With the warming of the atmospheric layer due to condensation and expansion of moist air with low pressure systems in the upper level of atmosphere, air mass system gains high speed motion in circulatory paths. It may be cyclonic

${ }^{*}$ Author for correspondence 
or anti-cyclonic, convergence or divergence, and linear transport system, depending on the local geography and its orientation such as mountains, valleys, and Iceland, according to various surfaces and their nature.

In general, Nepal receives about 2000-2500 mm rainfall in a year. About $80 \%$ of the total annual rainfall occurs during the summer monsoon season. ${ }^{1}$ Also, high rainfall variability has been observed in most part of the country. $\underline{2}$ The summer monsoon season exhibits large temporal and spatial variations with precipitation patterns governed by both monsoon circulation dynamics and orography. March is considered generally last of winter and beginning of the pre-monsoon periods in Nepal.

Winter source of moisture transport originates with the help of Westerly (air mass coming from the west), and if the moisture are from the Bay of Bengal, it is the transports from the South-East (SE). Winter moisture mass travel through long distance; therefore, it is drier in comparison to SE, which enter Nepal with the higher content of moisture in it (without rainfall in a short distance). The drier air masses (W) have less probability to rain easily in comparison to higher moisture in SE. Thus, contact of dry air mass with higher moist mass forms a boundary in which heat transformation or lightning takes place, for example, thunderstorms and hurricanes.

We have many experiences of thunderstorm, which generally don't have spinning wind leg/arm system as found in the case of tornadoes. Tornadoes are very large and strong atmospheric system, which develop due to large instability of atmospheric condition depending on very high wind speed and rotating on its axis that touch the earth surface. The convections of large air mass in the atmosphere, its circulation (vorticity) in its upper part and a rotating leg connection to the surface, is usually called vortex. In vortex arm, there is a huge force that can uplift even a large building or anything on its contact path. The speed (effect) of the leg arm is many times larger than the upper part, which simply looks like shape of an open umbrella.

\section{Material and Methods}

The size and speed of a tornado event generally vary with the heating system from the land, latent heat released within the atmosphere, altitude of local geography, and seasons and land or surface features.

Basic dynamical equations for a tornado system are as follows:
Pressure variation is controlled by: $[\delta p=-\rho g \delta z]$ Kinetic Energy applied in the system: $\left[\mathrm{KE}=\mathrm{mv}^{2} / 2\right]$ Centripetal force: $\left[\mathrm{CPF}=\mathrm{mv}^{2} / \mathrm{R}\right]$

Where, $\delta \mathrm{p}$ is the change in pressure; $\rho$ is the air density; $\mathrm{g}$ is the acceleration due to gravity $\delta \mathrm{z}$ is change of height or gradient height; $\mathrm{m}$ is the mass of the air including dust, etc.

$\mathrm{v}$ is the velocity of the air mass that becomes $(\mathrm{v} \operatorname{Cos} \theta)$ in the curvature flow and for a smaller change of $\theta$; $\operatorname{Cos}$ $\theta \approx 1$; $R$ is the radius of the curvature in which the air mass circulates around its systems. In the case of tornado formation, the forces outward and inward of circle of the curvature becomes zero, thus, the flow is controlled by $\delta p$ and $1 / 2^{*} m v^{2} \operatorname{Cos}^{2} \theta$. A simple diagram is shown (Figure 1) to elaborate force components under the cyclonic motion of such cyclones and the motion is controlled by pressure gradient force and Coriolis force component. In this study, the impacts of tornado were discussed based on the observation and literature review.

\section{Results and Discussion}

First Tornado Nepal (FTN) was observed to be of about $200 \mathrm{~km} \times 200 \mathrm{~km}$ in size and the leg spinning speeds were observed to be about $250 \mathrm{~km}$ ph, taking the mean value. The local eye sights explained, "there was a vertical funnel shaped structure together with its umbrella like tops rounded by thick black cloud," which is a true observation of a tornado, either small or big. Their observation proved that the wind speed was also very high, since the twisting leg arm lifted off many things along its path within about 10 to $20 \mathrm{~km}$; however, we consider that the maximum

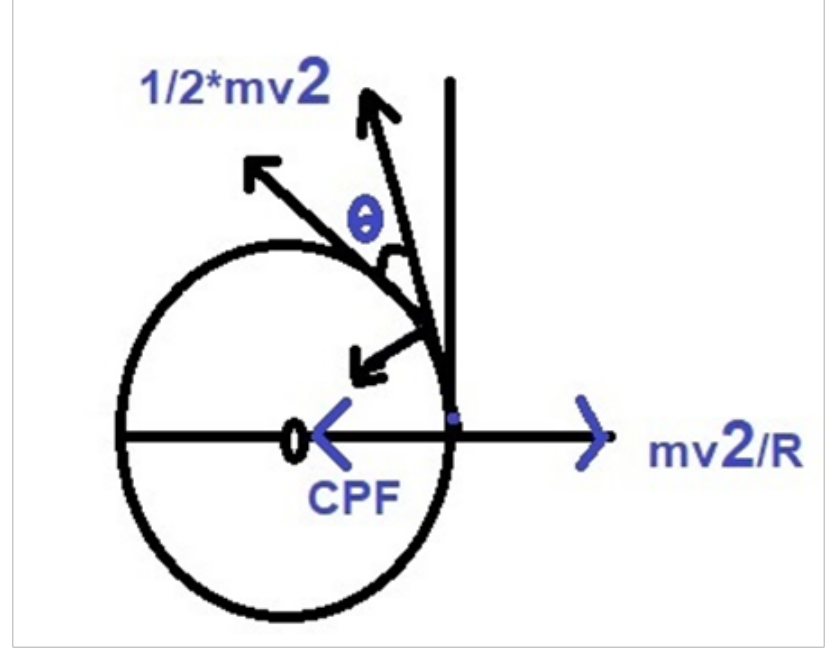

Figure 1. Mechanism for tornado formation. 
damages were found within Terai plain of about $(250$ $\mathrm{km} \max )$, since the tornado had a translation motion towards north from its origin near the border of Nepal. For reference, notable cell of the same FTN is depicted in Figures 2 and 3. It shows detection image of the first time tornado event in Nepal, which caused large destruction over the eastern Terai, Nepal.

Destructions by the FTN preliminary report indicated the damages of affected area $(20 \mathrm{sq} \mathrm{km})$ as: people dead: 28; injured: more than 1100; houses damaged: 2600; twisted trees: $15-20 \%$; and a few national parks and reserves and electric polls were also damaged. There could be still more unrecorded aspects from preliminary observation of FTN. So far, we have been observing more facts of FTN. Figure 3 shows more information behind its empowering development to carry out such unimagined destructions as mentioned in the previous studies. 4

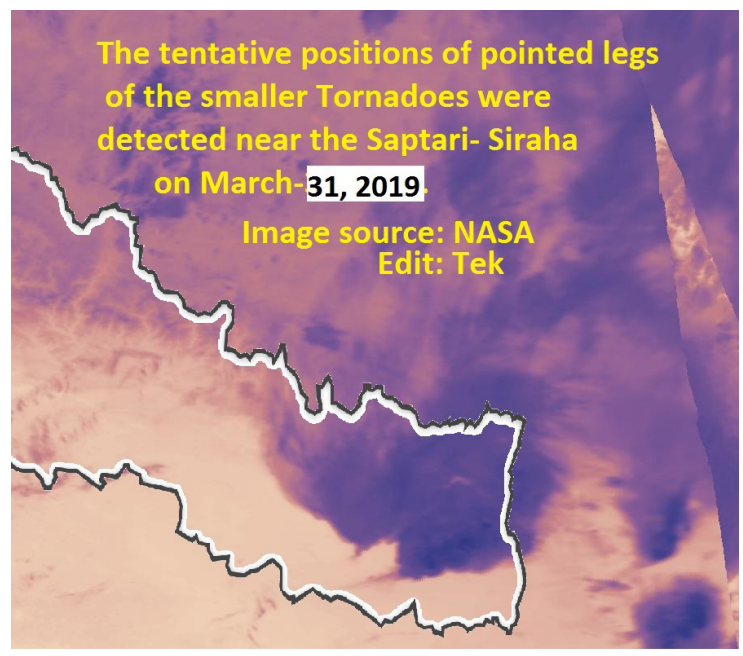

Figure 2. Detection of first tornado at eastern Terai, Nepal.

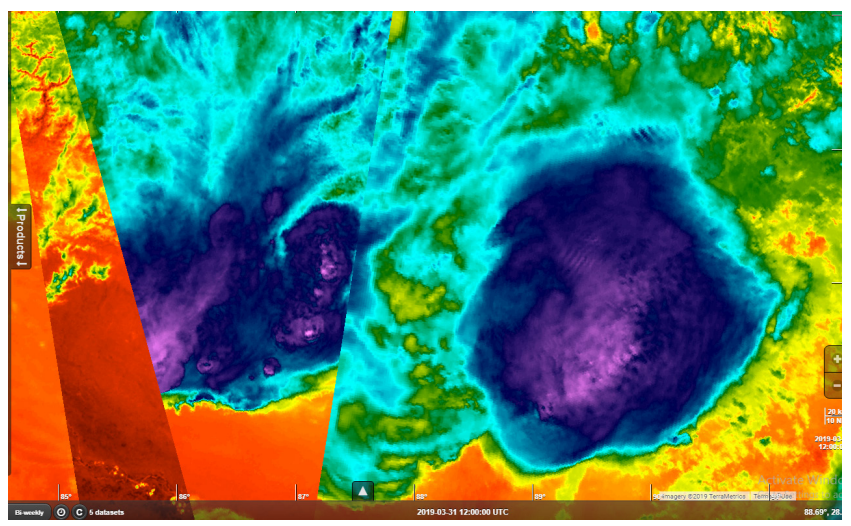

Figure 3. The size, position, and pointing funnel arms of tornado.
Further, we detected and confirmed the size, shapes, and position of FTN (Figures 2 and 3).

We see that there were actually two smaller tornadoes, spinning with their pointed legs-arms at two different places (Bara to Parsa) to the west from Janakpur. Among the two cells, one had double size than the other. The smaller cell was in the west, which could be developed due to westerly system (Figure 4), the same mass was found near Chitwan on 30 March, whereas the other cell might have developed from the air mass system that arrived from Bay of Bengal (Figure 4). The two cells are seen very close on 31 March 2019; this indicates they must be formed from different sources of air mass. As both of them were twisting very near and they joined into a single cell, they could have gained double power, which caused large destructions within 1-2 hours, later it might have lost its dynamic strength due to breakdown of spinning legs while entering the Siwalik or nearby mountain areas.

The FTN is recorded for the first time; however, there could be many such events in Nepal, where SE and Westerly monsoons provide high precipitation values in the southern slopes of the Himalayas, which could be affected due to long-term change in air mass circulations and temperature rise or other hidden climatic events such as typhoon or tornado in the high altitude Himalayas (Figure 5). Frequent monitoring and field observation studies lack in the high mountain areas of Nepal. FTN became public because it caused huge loss; if the same event had taken place somewhere in the Himalayas, nobody could have found it as the FTN. There are pre-records of torrential precipitation, flood hazards,

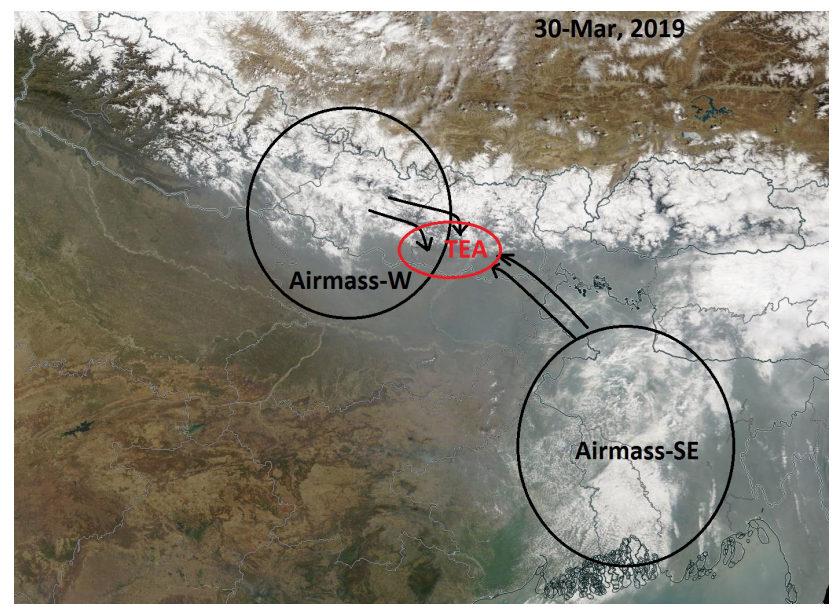

Figure 4. The air mass transportation from south east (SE) and west (W). 


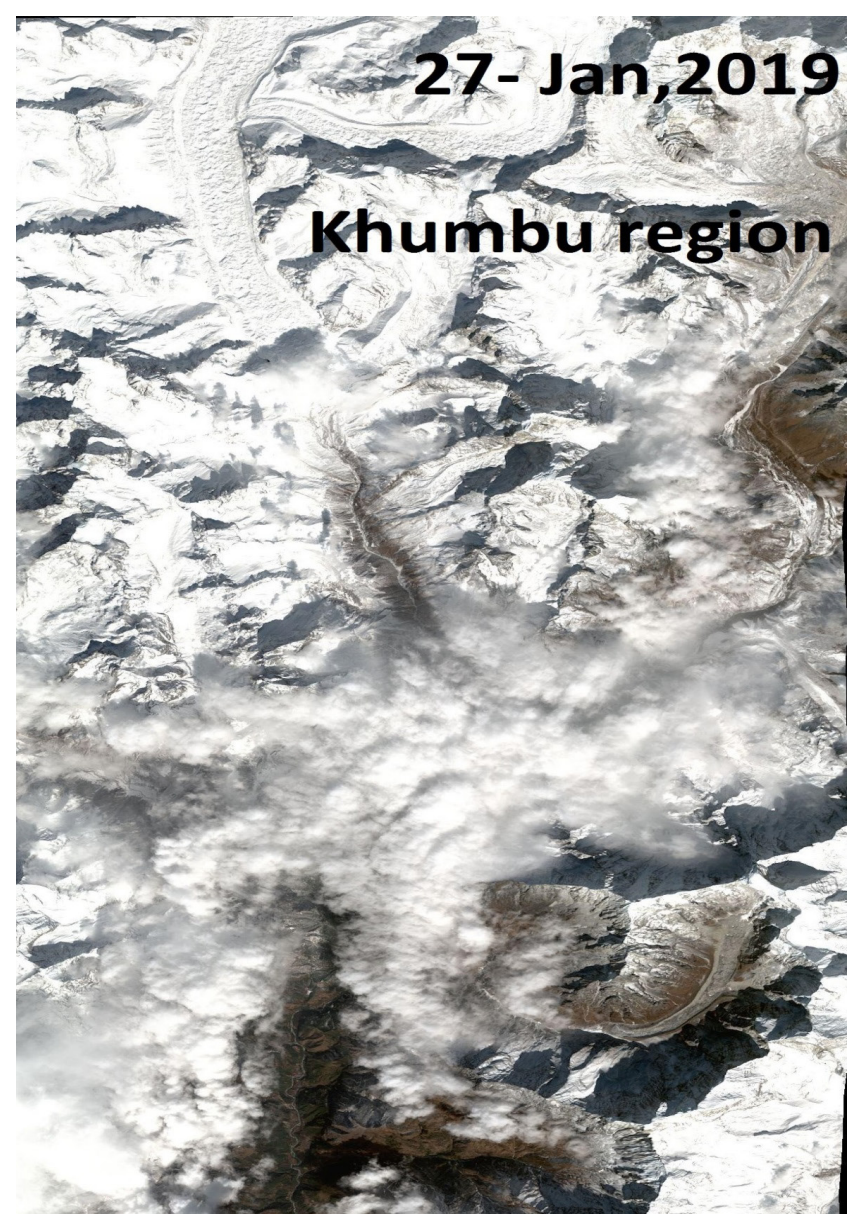

Figure 5. Small tornado in Khumbu area of Nepal.

landslides, and glacier lake outburst floods in different geographical points of Nepal, out of which some may be because of typhoons or tornadoes. Thus, we felt through this study that we really do not have the required modern detective equipment yet, which would help us infer before such an event like FTN. However, FTN will serve as a long-term memory and a lesson towards how to secure life of people and development process in various parts of Nepal.

The deadliest tornadoes striking in Asia have been found across a region that encompasses most of Bangladesh as well as in eastern states of India such as Assam, Tripura, West Bengal, and Orissa. $\underline{5}$ Two of recent tornadoes were found near the border area of India and Pakistan, which is shown in Figure 6.

These events were detected in May, which show the internal structures of both the tornadoes very clearly with circulating wind inside the cell structures. Both of these show their vortex arm touching the land surface

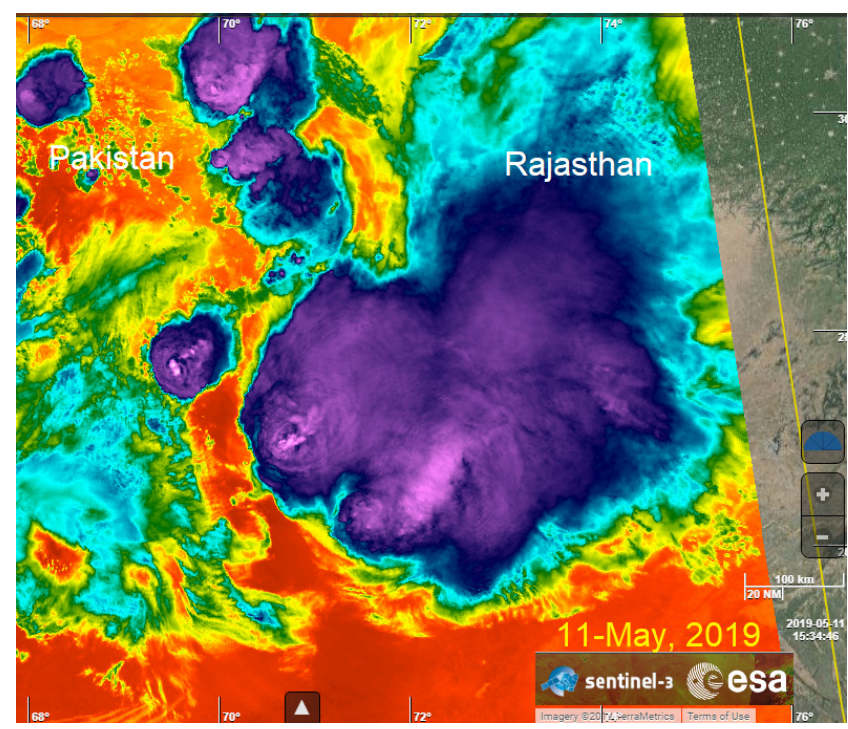

Figure 6. Tornadoes near the border area of India and Pakistan.

with opening various inlets of wind system into the storm-system. One of them shows a small plant like structure, which may be due to prestage of development process. The length of the vortex arms to the land is not seen as very long; however, the spinning upper part is interestingly seen very large showing a tendency to form a single cell within the very short time so far. The larger cell shows an opening mouth like structure just bending through neck like narrower of the funnel. Studying these internal structures of both tornadoes in Nepal, it is almost clear that the density of air mass is highly concentrated inside the funnel arms which varies with the length of arms in accordance to the altitudes variation. This high density might be due to vapour with dust and different garbage from the land surface. This informs that the length of the arm touching to the ground is higher for the case of large top part whirling air mass. In the case of FTN, it was considered that two of the air mass cyclonic system initially developed into one single cell just after breaking the leg-arm of the cell which may be due to opposite direction of the cyclones at the bottom part; thereafter its upper top part with similar direction of rotation with the other could have matched so that the power of the arm vortex became twice. Due to this reason the FTN made a large pick of many things on its ways even few houses and many plants were easily lifted and damaged. It is usually noted that the large warm inlayed dusty air in to the openings through the arm vortex in the case of their position on land system. From these 
image observations, it is clear that the sizes of tornadoes are smaller than the Japan, USA coastal areas tornadoes and they are formed due to long term instability of the tropospheric atmosphere particularly in the dry periods in pre-monsoon season.

However, the information makes difficult for the average annual number of tornadoes in the region. Most tornadoes occur there from early April to March and sometimes in late May. Most violent tornadoes occurred in the afternoon and evening.

Reports of tornadoes have also come from several other Asian nations, including Japan, Phillippines, Vietnam, Myanmar, and Pakistan. Most of the tornadoes in Japan occur from July to October and frequently in September.

\section{Conclusion and Recommendation}

In sum, more powerful and dangerous tornadoes are possible in the southern slope of Himalayas where influences of monsoon circulation are large particularly at different parts of Nepal, India, and Bangladesh. Regional climate change has been showing its newer interactions and enhancing larger impacts on various environments in south Asia. Department of Hydrology and Meteorology, Nepal Government, has been measuring the data and also initiated some preventing measures in the field of such disasters every year such as monsoon flooding and other various kinds of natural disasters; however, there is still lack of installation of modern scientific equipment at different parts of the country, empowering high skilled scientists and training opportunities to younger officers in order to make them capable for timely forecasting such events and make the society better secured. Without the advance research facilities of regional weather and climate change South Asia won't be free from climatic disasters to live.

\section{Conflict of Interest}

The authors declare that they have no conflict of interest.

\section{Acknowledgements}

This research was supported by the President's International Fellowship Initiative (PIFI) program of Chinese Academy of Sciences for the first Author (TBC). We would like to thank Institute of Tibetan plateau Research, Chinese Academy of Sciences for providing the good working platform in this topic. We also extend our acknowledgements to the NASA and Eumetsat Organization for their real-time images from the online free pages. The corresponding author (BD) is thankful to Kathmandu Center for Research and Education, Chinese Academy of Sciences-Tribhuvan University, (KCRE) for the support.

\section{References}

1. Shrestha ML. Interannual variation of summer monsoon rainfall over Nepal and its relation to Southern Oscillation Index. Meteorol Atmos Phys. 2000;75:21-8.

2. Dhital YP, Kayastha RB. Frequency analysis causes and impacts of flooding in the Bagmati River Basin, Nepal. J Flood Risk Manag. 2013;6:253-60.

3. Malik N, Marwan N, Kurths J. Spatial structures and directionalities in monsoonal precipitation over South Asia. Nonlinear Process Geophys. 2010;17:371-81.

4. Nepali scientists record country's first tornado. [cited 2019 Apr 12]. https://www.nature.com/articles/d41586-01901159-w.

5. Bangladesh and East India tornado prediction site. [cited 2019]. https://www.bangladeshtornadoes.org/. 